\title{
Hydrogen Sulfide is Involved in the Regulation of Ascorbate-glutathione Cycle by Exogenous ABA in Wheat Seedling Leaves under Osmotic Stress
}

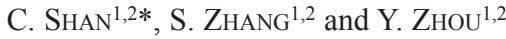 \\ ${ }^{1}$ Henan Institute of Science and Technology, Xinxiang, 453003, China \\ ${ }^{2}$ Collaborative Innovation Center of Modern Biological Breeding, Henan Province, Xinxiang, 453003, China
}

(Received 29 September 2016; Accepted 6 December 2016;

Communicated by A. Pécsváradi)

\begin{abstract}
This study investigated the role of hydrogen sulfide $\left(\mathrm{H}_{2} \mathrm{~S}\right)$ in the regulation of ascorbateglutathione (AsA-GSH) cycle by exogenous ABA in wheat leaves under osmotic stress. The results showed that osmotic stress significantly increased the activities of ascorbate peroxidase (APX), glutathione reductase (GR), monodehydroascorbate reductase (MDHAR) and dehydroascorbate reductase (DHAR), the ratio of reduced ascorbate to oxidized ascorbate (AsA/DHA) and reduced glutathione to oxidized glutathione (GSH/GSSG), the malondialdehyde content and electrolyte leakage, and the $\mathrm{H}_{2} \mathrm{~S}$ content, compared to control. Exogenous ABA significantly increased above indicators under osmotic stress, compared to osmotic stress alone. Above activity increases except MDHAR activity were suppressed by application of $\mathrm{H}_{2} \mathrm{~S}$ scavenger hypotaurine (HT) and synthesis inhibitor aminooxyacetic acid (AOA). Meanwhile, exogenous ABA significantly decreased malondialdehyde content and electrolyte leakage induced by osmotic stress. Application of HT and AOA reversed above effects of application of exogenous ABA. Application of NaHS can reversed above effects of $\mathrm{HT}$ and AOA. Our results suggested that $\mathrm{H}_{2} \mathrm{~S}$ induced by exogenous ABA is a signal that leads to the up-regulation of AsA-GSH cycle.
\end{abstract}

Keywords: polyethylene glycol, sodium hydrosulfide, oxidative damage, Triticum aestivum, abscisic acid

\section{Introduction}

Osmotic stress adversely affects plant growth and productivity (Golldack et al. 2011). Osmotic stress usually causes the overproduction of reactive oxygen species (ROS), which result in oxidative damage to plants (Apel and Hirt 2004). In order to protect themselves from oxidative damage, plants could enhance the ascorbate-glutathione (AsAGSH) cycle (Shan et al. 2015; Dai et al. 2015). Through this cycle, two important antioxidants ascorbate (AsA) and glutathione (GSH) are regenerated and $\mathrm{H}_{2} \mathrm{O}_{2}$ is scavenged (Shan and Liang 2010). Thus, the AsA-GSH cycle plays an important role in limiting oxidative damage by maintaining the contents of ascorbate and glutathione and respective redox states in plants.

*Corresponding author; E-mail: shchjuan1978@aliyun.com 
Abscisic acid (ABA) is an important plant hormone that plays important roles in regulating stress responses (Gupta et al. 2012; Parvin et al. 2012). Increasing evidence shows that ABA serves an important role in limiting oxidative damage by enhancing AsA-GSH cycle in plants (Zhang et al. 2007). It has been reported that signal molecules $\mathrm{NO}, \mathrm{H}_{2} \mathrm{O}_{2}$, $\mathrm{Ca}^{2+}-\mathrm{CaM}$ and MAPK participate in the process of ABA signal transduction in regulating the ascorbate-glutathione cycle (Zhang et al. 2007; Hu et al. 2007). Hydrogen sulfide $\left(\mathrm{H}_{2} \mathrm{~S}\right)$ is an important gaseous signaling molecule in plants (Hancock and Whiteman 2014). Zhang et al. (2008) proved that exogenous $\mathrm{H}_{2} \mathrm{~S}$ affected the antioxidative response against osmotic stress. Our previous study found that exogenous $\mathrm{H}_{2} \mathrm{~S}$ regulated AsA-GSH cycle in wheat leaves under osmotic stress (Shan et al. 2011). Liu et al. (2011) have reported that endogenous $\mathrm{H}_{2} \mathrm{~S}$ is involved in the process of ABA-induced stomatal closure. However, whether endogenous $\mathrm{H}_{2} \mathrm{~S}$ participates in the regulation of AsA-GSH cycle by ABA in plants under osmotic stress remains unknown. To describe antioxidant mechanisms of plants, the role of $\mathrm{H}_{2} \mathrm{~S}$ in the regulation of AsA-GSH cycle by ABA under osmotic stress deserves further consideration.

This study investigated malondialdehyde (MDA) content, electrolyte leakage, the activities of enzymes in AsA-GSH cycle, the ratios of AsA/DHA and GSH/GSSG in the leaves of Jimai 21 seedlings exposed to osmotic stress induced by $15 \%$ polyethylene glycol (PEG)-6000. The specific objective of the study was to describe the role of $\mathrm{H}_{2} \mathrm{~S}$ in the regulation of AsA-GSH cycle by ABA in wheat plants under osmotic stress by using $\mathrm{H}_{2} \mathrm{~S}$ scavenger hypotaurine (HT), $\mathrm{H}_{2} \mathrm{~S}$ synthesis inhibitor aminooxyacetic acid (AOA) and $\mathrm{H}_{2} \mathrm{~S}$ donor NaHS.

\section{Materials and Methods}

\section{Plant culture and treatment}

Wheat (Triticum aestivum L., cv. Jimai 21) seeds were sown in plastic trays filled with a sand/vermiculite matter mix $(2: 1, \mathrm{v} / \mathrm{v})$ and grown in a greenhouse. Culture conditions: $25 / 15^{\circ} \mathrm{C}$ (day/night) temperature and $500 \mu \mathrm{mol} \mathrm{m} \mathrm{m}^{-2} \mathrm{~s}^{-1}$ light intensity with a $12 \mathrm{~h}$ photoperiod. The seedlings were watered with half-strength Hoagland's solution every day. Seedlings of uniform height were selected when the third leaf was fully expanded. The roots of selected plants were washed and placed in beakers containing $50 \mathrm{ml} \mathrm{15 \%}(\mathrm{w} / \mathrm{v})$ PEG solution for 24 and $48 \mathrm{~h}$ under above conditions after soaking in half-strength Hoagland's solution for $12 \mathrm{~h}$. The beakers were wrapped with aluminium foil to keep roots in dark. To study the effect of ABA, the roots of plants were soaked in $100 \mu \mathrm{M}$ ABA solution for $8 \mathrm{~h}$ and then exposed to PEG solution for 24 and $48 \mathrm{~h}$. To study the effects of HT and AOA, the roots of plants were soaked in $20 \mu \mathrm{M}$ HT or $0.3 \mathrm{mM}$ AOA or $100 \mu \mathrm{M}$ $\mathrm{ABA}+20 \mu \mathrm{M}$ HT or $100 \mu \mathrm{M} \mathrm{ABA}+0.3 \mathrm{mM}$ AOA solution for $8 \mathrm{~h}$ and then exposed to PEG solution for 24 and $48 \mathrm{~h}$. To investigate whether the effects of HT and AOA can be reversed by exogenous $\mathrm{H}_{2} \mathrm{~S}$, the roots of plants were soaked in $20 \mu \mathrm{M}$ HT or $0.3 \mathrm{mM}$ $\mathrm{AOA}$ or $100 \mu \mathrm{M} \mathrm{ABA}+20 \mu \mathrm{M}$ HT or $100 \mu \mathrm{M} \mathrm{ABA}+0.3 \mathrm{mM}$ AOA solution for $8 \mathrm{~h}$ and then exposed to $\mathrm{NaHS}\left(\mathrm{H}_{2} \mathrm{~S}\right.$ donor $)+15 \%$ PEG or half-strength Hoagland's solution for 
24 and $48 \mathrm{~h}$. Above solutions used to treat plants were prepared by adding corresponding substances into half-strength Hoagland's solution. Control plants were treated with halfstrength Hoagland's solution alone. Following 24 and $48 \mathrm{~h}$ treatment, the third fully-expanded leaf of wheat seedlings was collected and immediately frozen in liquid nitrogen, and stored at $-80{ }^{\circ} \mathrm{C}$ until analyses.

\section{The extraction and assay of enzymes in AsA-GSH cycle}

Enzymes were extracted according to Shan and Liang (2010). The activities of ascorbate peroxidase (APX, EC 1.11.1.11), glutathione reductase (GR, EC 1.6.4.2), monodehydroascorbate reductase (MDHAR, EC 1.6.5.4) and dehydroascorbate reductase (DHAR, EC 1.8.5.1) were measured according to Nakano and Asada (1981), Grace and Logan (1996), Miyake and Asada (1992) and Dalton et al. (1986), respectively. The specific enzyme activity for all the above enzymes was expressed as units $\mathrm{mg}^{-1}$ protein. Protein concentration was measured according to Bradford (1976).

\section{Determination of AsA, DHA, GSSG and GSH}

The contents of AsA, DHA, GSSG and GSH were measured according to Hodges et al. (1996) and Griffith (1980), respectively. AsA/DHA was expressed as the ratio between the content of AsA and the content of DHA. GSH/GSSG was expressed as the ratio between the content of GSH and the content of GSSG.

\section{Determination of $\mathrm{H}_{2} \mathrm{~S}, \mathrm{MDA}$ and electrolyte leakage}

$\mathrm{H}_{2} \mathrm{~S}$ content was determined by formation of methylene blue from dimethyl- $p$-phenylenediamine in $\mathrm{H}_{2} \mathrm{SO}_{4}$ as described previously (Zhang et al. 2008). MDA content was measured according to Hodges et al. (1999). Electrolyte leakage was determined according to Zhao et al. (2004).

\section{Statistical analysis}

Data presented were the mean values of five times with four seedlings each time. Statistical assays were carried out by one-way analysis of variance and Duncan's multiple range test at the $5 \%$ level of significance.

\section{Results}

\section{Effect of exogenous ABA, HT, AOA and NaHS on $\mathrm{H}_{2} \mathrm{~S}$ content}

Osmotic stress led to an increase in $\mathrm{H}_{2} \mathrm{~S}$ content, compared with control (Fig. 1). After 24 and $48 \mathrm{~h}$ of treatment, osmotic stress increased $\mathrm{H}_{2} \mathrm{~S}$ content by $123.3 \%$ and $80 \%$, respectively. Exogenous ABA significantly induced the production of $\mathrm{H}_{2} \mathrm{~S}$ in stressed leaves, 
compared with osmotic stress alone. After 24 and $48 \mathrm{~h}$ of treatment, exogenous ABA increased $\mathrm{H}_{2} \mathrm{~S}$ content by $50 \%$ and $46.7 \%$ under osmotic stress, respectively. ABA-pretreatment alone also increased $\mathrm{H}_{2} \mathrm{~S}$ content, compared with the control. Pretreatments with HT and AOA significantly lowered the accumulation of $\mathrm{H}_{2} \mathrm{~S}$ during osmotic stress when applied alone or in conjunction with ABA. The addition of NaHS reversed the effects of HT and $\mathrm{AOA}$ on the accumulation of $\mathrm{H}_{2} \mathrm{~S}$ during osmotic stress when applied alone or in conjunction with ABA.

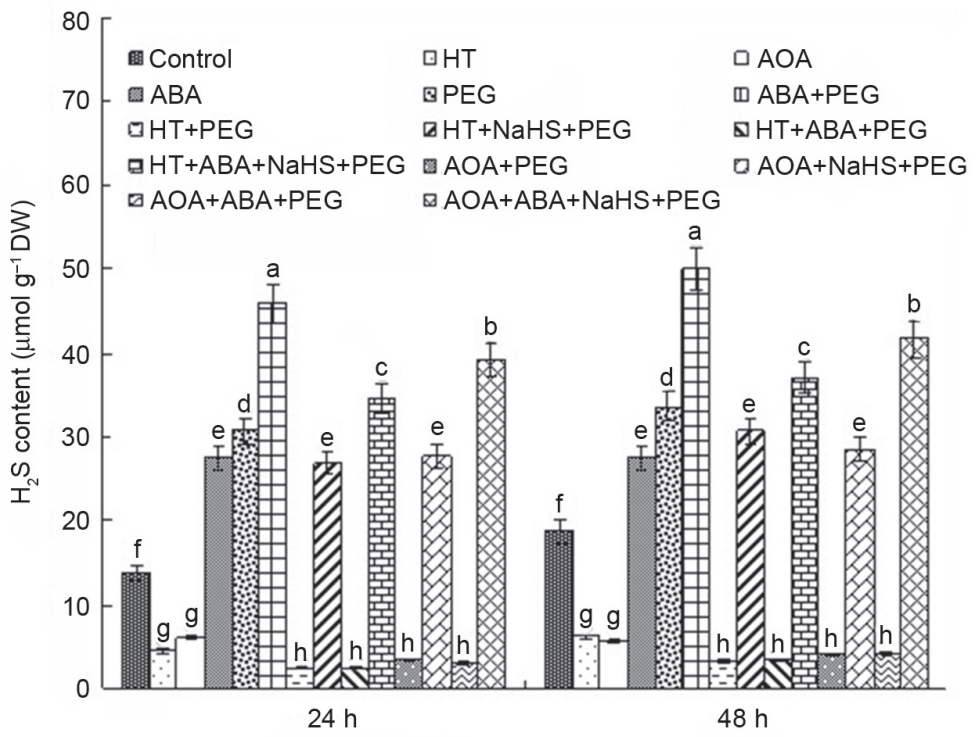

Figure 1. Effects of exogenous ABA, HT, AOA and NaHS on $\mathrm{H}_{2} \mathrm{~S}$ content in leaves of PEG-induced osmotic stressed leaves. The plants were treated as follows: Control, half-strength Hoagland's solution; HT, $20 \mu \mathrm{M} \mathrm{HT}$; AOA, 0.3 mM AOA; ABA, $100 \mu \mathrm{M}$ ABA; PEG, 15\% PEG; ABA+PEG, $100 \mu \mathrm{M}$ ABA+ 15\% PEG; HT+PEG, $20 \mu \mathrm{M} \mathrm{HT}+15 \%$ PEG; HT+NaHS + PEG, $20 \mu \mathrm{M}$ HT + $0.3 \mathrm{mM} \mathrm{NaHS}+15 \%$ PEG; HT +ABA+PEG, $20 \mu \mathrm{M}$ $\mathrm{HT}+100 \mu \mathrm{M} \mathrm{ABA}+15 \% \mathrm{PEG} ; \mathrm{HT}+\mathrm{ABA}+\mathrm{NaHS}+\mathrm{PEG}, 20 \mu \mathrm{M} \mathrm{HT}+100 \mu \mathrm{M} \mathrm{ABA}+0.3 \mathrm{mM} \mathrm{NaHS}+15 \%$ PEG; AOA + PEG, $0.3 \mathrm{mM} \mathrm{AOA}+15 \% \mathrm{PEG}$; AOA + NaHS + PEG, $0.3 \mathrm{mM} \mathrm{AOA}+0.3 \mathrm{mM} \mathrm{NaHS}+15 \% \mathrm{PEG}$; $\mathrm{AOA}+\mathrm{ABA}+\mathrm{PEG}, 0.3 \mathrm{mM} \mathrm{AOA}+100 \mu \mathrm{M} \mathrm{ABA}+15 \% \mathrm{PEG} ; \mathrm{AOA}+\mathrm{ABA}+\mathrm{NaHS}+\mathrm{PEG}, 0.3 \mathrm{mM} \mathrm{AOA}+100$ $\mu \mathrm{M} \mathrm{ABA}+0.3 \mathrm{mM} \mathrm{NaHS}+15 \%$ PEG. The plants were pretreated with ABA or HT or AOA or ABA $+\mathrm{HT}$ or $\mathrm{ABA}+\mathrm{AOA}$ for $8 \mathrm{~h}$, and then exposed to $15 \% \mathrm{PEG}$ or NaHS $+15 \%$ PEG for 24 and $48 \mathrm{~h}$. Values represent mean \pm standard deviations (SD), small letters stand for significant difference among different treatments at $\mathrm{P}<0.05$

Effects of exogenous ABA, HT, AOA and NaHS on the activities of enzymes in AsA-GSH cycle

Osmotic stress significantly increased the activities of APX, GR, DHAR and MDHAR, compared with control (Fig. 2). After 24 h of treatment, osmotic stress increased the activities of APX, GR, DHAR and MDHAR by $133.3 \%, 158.3 \%, 130.8 \%$ and $100 \%$, respectively. After $48 \mathrm{~h}$ of treatment, osmotic stress increased the activities of APX, GR, 

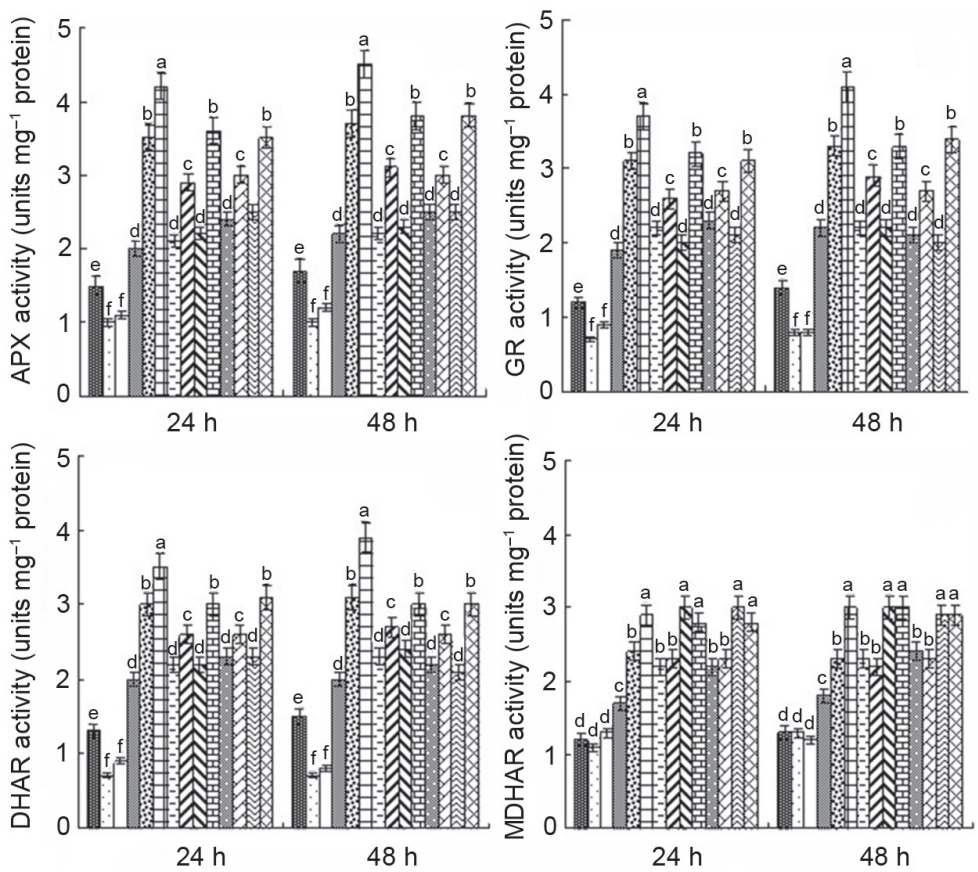

Figure 2. Effects of exogenous ABA, HT, AOA and NaHS on the activities of APX (A), GR (B), DHAR (C) and MDHAR (D) in PEG-induced osmotic stressed leaves. The plants were treated as described in the legend of Fig. 1

DHAR and MDHAR by $117.6 \%, 135.7 \%, 100 \%$ and $76.9 \%$, respectively. Treatment with ABA before applying PEG significantly increased the activities of above enzymes, compared with osmotic stress alone. After $24 \mathrm{~h}$ of treatment, treatment with ABA before applying PEG increased the activities of APX, GR, DHAR and MDHAR by $20 \%, 19.3 \%$, $16.7 \%$ and $20.8 \%$, respectively. After $48 \mathrm{~h}$ of treatment, treatment with ABA before applying PEG increased the activities of APX, GR, DHAR and MDHAR by $21.6 \%, 24.2 \%$, $30 \%$ and $17.4 \%$, respectively. ABA-pretreatment alone also increased the activities of APX, GR, DHAR and MDHAR, compared with the control. HT and AOA significantly reduced the activities of APX, GR, DHAR but not MDHAR during osmotic stress with or without ABA. The addition of NaHS reversed above effects of HT and AOA on the activities of APX, GR and DHAR.

\section{Effects of exogenous ABA, HT, AOA and NaHS on the redox state of AsA and GSH}

Osmotic stress significantly decreased the ratios of AsA/DHA and GSH/GSSG, compared with control (Fig. 3). After $48 \mathrm{~h}$ of treatment, osmotic stress decreased the ratios of AsA/ DHA and GSH/GSSG by $36.8 \%$ and $35 \%$, respectively. After $48 \mathrm{~h}$ of treatment, osmotic stress decreased the ratios of AsA/DHA and GSH/GSSG by $45 \%$ and $42.8 \%$, respectively. 

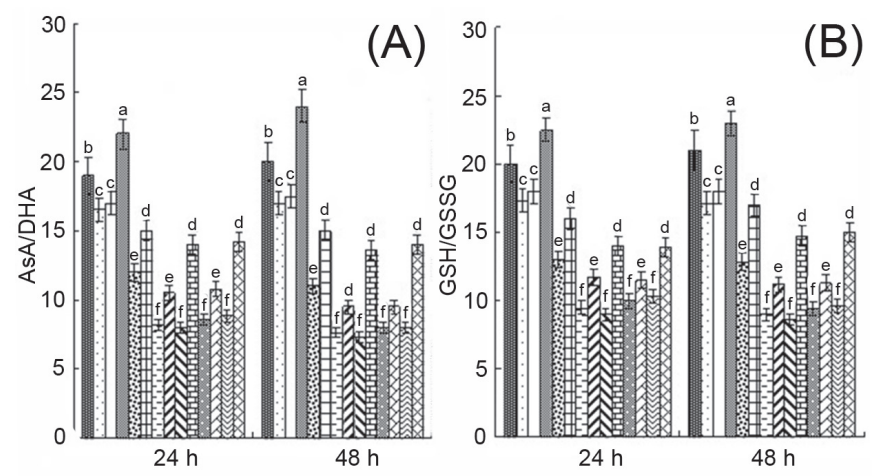

Figure 3. Effects of exogenous ABA, HT, AOA and NaHS on the ratios of AsA/DHA (A) and GSH/GSSG (B) in PEG-induced osmotic stressed leaves. The plants were treated as described in the legend of Fig. 1

Treatment with ABA before applying PEG significantly increased AsA/DHA and GSH/ GSSG ratios, compared with osmotic stress alone. After $24 \mathrm{~h}$ of treatment, treatment with ABA before applying PEG increased the ratios of AsA/DHA and GSH/GSSG by $25 \%$ and $23.1 \%$, respectively. After $48 \mathrm{~h}$ of treatment, treatment with ABA before applying PEG increased the ratios of AsA/DHA and GSH/GSSG by $36.4 \%$ and $41.6 \%$, respectively. ABA-pretreatment alone also increased the ratios of AsA/DHA and GSH/GSSG, compared with the control. HT and AOA significantly reduced AsA/DHA and GSH/GSSG ratios induced by osmotic stress with or without ABA. The addition of NaHS reversed above effects of HT and AOA on AsA/DHA and GSH/GSSG ratios induced by osmotic stress with or without ABA.

\section{Effects of exogenous ABA, HT, AOA and NaHS on MDA content and electrolyte leakage}

Osmotic stress led to increases in MDA content and electrolyte leakage, compared with control (Fig. 4). After $24 \mathrm{~h}$ of treatment, osmotic stress increased MDA content and electrolyte leakage by $147.8 \%$ and $200 \%$, respectively. After $48 \mathrm{~h}$ of treatment, osmotic stress increased MDA content and electrolyte leakage by $143.6 \%$ and $125 \%$, respectively. Treatment with ABA before applying PEG significantly reduced the MDA content and electrolyte leakage, compared with osmotic stress alone. After $24 \mathrm{~h}$ of treatment, treatment with ABA before applying PEG decreased MDA content and electrolyte leakage by $26.3 \%$ and $37.5 \%$, respectively. After $48 \mathrm{~h}$ of treatment, treatment with ABA before applying PEG decreased MDA content and electrolyte leakage by $40.3 \%$ and $33.3 \%$, respectively. ABApretreatment alone had no obvious effect on MDA content and electrolyte leakage, compared with the control. Pretreatments with HT and AOA significantly increased the MDA content and electrolyte leakage of stressed leaves, compared with osmotic stress with or without ABA. The addition of NaHS reversed above effects of HT and AOA on MDA content and electrolyte leakage induced by osmotic stress with or without ABA. 

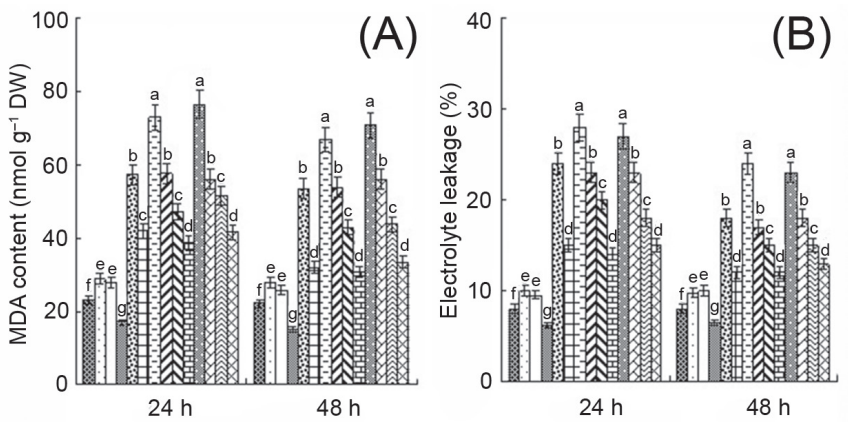

Figure 4. Effects of exogenous ABA, HT, AOA and NaHS on MDA content (A) and electrolyte leakage (B) of PEG-induced osmotic stressed leaves. The plants were treated as described in the legend of Fig. 1

\section{Discussion}

Many studies showed that osmotic stress induced oxidative damage in plants. In the current study, higher levels of lipid peroxidation, as indicated by MDA content and electrolyte leakage, was observed in wheat leaves in response to osmotic stress. To cope with the oxidative stress induced by osmotic stress, the activity of the AsA-GSH cycle in wheat leaves was up-regulated. It has been reported that exogenous ABA will up-regulate the activity of the AsA-GSH cycle in maize leaves through APX and GR activity, which alleviates the oxidative stress induced by osmotic stress (Zhang et al. 2007; Hu et al. 2007). In the present study, it was also found that exogenous ABA enhanced the activities of APX and GR under osmotic stress. Besides, this study found that exogenous ABA could increase the activities of DHAR and MDHAR involved in the AsA-GSH cycle in wheat leaves under osmotic stress.

AsA and GSH are major redox compounds in plants. It has been reported that the changes in the ratios of AsA/DHA and GSH/GSSG are more important than the individual changes in AsA and GSH content in response to oxidative stress (Kocsy et al. 2001). Our results showed that osmotic stress significantly decreased the ratios of AsA/DHA and GSH/GSSG. Osmotic stress plus ABA significantly increased AsA/DHA and GSH/GSSG ratios, compared with osmotic stress alone. Besides, our results indicated that signal molecular $\mathrm{H}_{2} \mathrm{~S}$ participated in the up-regulation of AsA/DHA and GSH/GSSG ratios by ABA under osmotic stress. Liu et al. (2011) reported that endogenous $\mathrm{H}_{2} \mathrm{~S}$ is involved in the process of ABA-induced stomatal closure. Present study showed that $\mathrm{H}_{2} \mathrm{~S}$ induced by exogenous ABA and osmotic stress was involved in the regulation of AsA-GSH cycle in wheat leaves through APX, GR and DHAR, which resulted in the increases in the ratios of AsA/DHA and GSH/GSSG under osmotic stress without or plus ABA. Christou et al. (2013) reported that $\mathrm{H}_{2} \mathrm{~S}$ could maintain high ascorbate and glutathione redox states through APX and GR in the AsA-GSH cycle under osmotic stress, which was consistent with our results. These results suggested that $\mathrm{H}_{2} \mathrm{~S}$ served an important role in ABA signaling and in the regulation of AsA-GSH cycle of plants. 
It has been shown that osmotic stress and exogenous ABA can induce the production of $\mathrm{H}_{2} \mathrm{~S}$ and that endogenous $\mathrm{H}_{2} \mathrm{~S}$ is involved in the process of ABA-induced stomatal closure (Liu et al. 2011; Wang et al. 2012). Similar results were found in the present study, exogenous ABA and osmotic stress also could induce the production of endogenous $\mathrm{H}_{2} \mathrm{~S}$. Besides, the results of our present study found that $\mathrm{H}_{2} \mathrm{~S}$ induced by exogenous ABA and osmotic stress was involved in the regulation of AsA-GSH cycle in wheat leaves through APX, GR and DHAR, which, in turn, increased the ratios of AsA/DHA and GSH/GSSG under osmotic stress alone and osmotic stress plus ABA. These results suggested that $\mathrm{H}_{2} \mathrm{~S}$ served an important role in ABA signaling and in the regulation of AsA-GSH cycle of plants.

As a stress-signaling molecule, ABA serves important roles in defending against oxidative stress in plant cells (Ding et al. 2009). Hu et al. (2008) has reported that NO, MAPK and $\mathrm{H}_{2} \mathrm{O}_{2}$ are all involved in the regulation of AsA-GSH cycle by ABA under osmotic stress. In the present study, $\mathrm{H}_{2} \mathrm{~S}$ was also involved in the regulation of AsA-GSH cycle by ABA under osmotic stress. However, whether there is relationship between NO, MAPK, $\mathrm{H}_{2} \mathrm{O}_{2}$ and $\mathrm{H}_{2} \mathrm{~S}$ in the regulation of AsA-GSH cycle in ABA signaling remains unknown. Further studies should investigate the relationship between $\mathrm{NO}, \mathrm{MAPK}, \mathrm{H}_{2} \mathrm{O}_{2}$ and $\mathrm{H}_{2} \mathrm{~S}$ in the regulation of AsA-GSH cycle in ABA signaling to provide more knowledge for the antioxidant metabolism in plants under osmotic stress.

In conclusion, the results clearly suggest that exogenous ABA and osmotic stress-induced $\mathrm{H}_{2} \mathrm{~S}$ accumulation participates in the regulation of AsA-GSH cycle, which, in turn, enhances the antioxidant ability and protects wheat seedling against oxidative stress induced by osmotic stress. These results provide new knowledge to the antioxidant metabolism in plants under osmotic stress.

\section{Acknowledgement}

This study was funded by Open project of Crop Science Characteristic Discipline of Henan Province, The National Natural Science Foundation of China (31571667 and U1204315).

\section{References}

Apel, K., Hirt, H. 2004. Reactive oxygen species: Metabolism, oxidative stress, and signal transduction. Annu. Rev. Plant Biol. 55:373-399.

Bradford, M.M. 1976. A rapid and sensitive method for the quantitation of microgram quantities of protein utilizing the principle of protein-dye binding. Anal. Biochem. 72:248-254.

Christou, A., Manganaris, G.A., Papadopoulos, I., Fotopoulos, V. 2013. Hydrogen sulfide induces systemic tolerance to salinity and non-ionic osmotic stress in strawberry plants through modification of reactive species biosynthesis and transcriptional regulation of multiple defence pathways. J. Exp. Bot. 64:19531966.

Dai, H., Jia, G., Shan, C. 2015. Jasmonic acid-induced hydrogen peroxide activates MEK1/2 in up-regulating the redox states of ascorbate and glutathione in wheat leaves. Acta Physiol. Plant. 37:200. 
Dalton, D.A., Russell, S.A., Hanus, F.J., Pascoe, G.A., Evans, H.J. 1986. Enzymatic reactions of ascorbate and glutathione that prevent peroxide damage in soybean root nodules. Proc. Natl. Acad. Sci. USA 83:38113815 .

Ding, H.D., Zhang, A.Y., Wang, J.X., Lu, R., Zhang, H., Zhang, J.H., Jiang, M.Y. 2009. Identity of an ABAactivated $46 \mathrm{kDa}$ mitogen-activated protein kinase from Zea mays leaves: Partial purification, identification and characterization. Planta 230:239-251.

Golldack, D., Lüking, I., Yang, O. 2011. Plant tolerance to drought and salinity: Stress regulating transcription factors and their functional significance in the cellular transcriptional network. Plant Cell Rep. 30:13831391.

Grace, S.C., Logan, B.A. 1996. Acclimation of foliar antioxidant systems to growth irradiance in three broadleaved evergreen species. Plant Physiol. 112:1631-1640.

Griffith, O.W. 1980. Determination of glutathione and glutathione disulfide using glutathione reductase and 2-vinylpyridine. Anal. Biochem. 106:207-212.

Gupta, K., Gupta, B., Ghosh, B., Sengupta, D. 2012. Spermidine and abscisic acid-mediated phosphorylation of a cytoplasmic protein from rice root in response to salinity stress. Acta Physiol. Plant. 34:29-40.

Hancock, J.T., Whiteman, M. 2014. Hydrogen sulfide and cell signaling: Team player or referee? Plant Physiol. Biochem. 78:37-42.

Hodges, M.D., DeLong, J.M., Forney, C.F., Prange, R.K. 1999 Improving the thiobarbituric acid-reactivesubstances assay for estimating lipid peroxidation in plant tissues containing anthocyanin and other interfering compounds. Planta 207:604-611.

Hodges, D.M., Andrews, C.J., Johnson, D.A., Hamilton, R.I. 1996. Antioxidant compound responses to chilling stress in differentially sensitive inbred maize lines. Plant Physiol. 98:685-692.

Hu, X., Jiang, M., Zhang, J., Zhang, A., Lin, F., Tan, M. 2007. Calcium-calmodulin is required for abscisic acid-induced antioxidant defense and functions both upstream and downstream of $\mathrm{H}_{2} \mathrm{O}_{2}$ production in leaves of maize (Zea mays) plants. New Phytol. 173:27-38.

Hu, X., Wang, W., Li, C., Zhang, J., Lin, F., Zhang, A., Jiang, M. 2008. Cross-talks between $\mathrm{Ca}^{2+} / \mathrm{CaM}$ and $\mathrm{H}_{2} \mathrm{O}_{2}$ in abscisic acid-induced antioxidant defense in leaves of maize plants exposed to water stress. Plant Growth Regul. 55:183-198.

Kocsy, G., Galiba, G., Brunold, C. 2001. Role of glutathione in adaptation and signaling during chilling and cold acclimation in plants. Physiol. Plant. 113:158-164.

Liu, J., Hou, Z., Zhao, F., Liu, X. 2011. Hydrogen sulfide mediates ABA-induced stomatal closure of Vicia faba L. Acta Bot. Boreal-Occident Sin. 31:298-304. (in Chinese)

Miyake, C., Asada, K. 1992. Thylakoid-bound ascorbate peroxidase in spinach chloroplasts and photoreduction of its primary oxidation product monodehydroascorbate radicals in thylakoids. Plant Cell Physiol. 33:541553.

Nakano, Y., Asada, K. 1981. Hydrogen peroxide is scavenged by ascorbate specific peroxidase in spinach chloroplasts. Plant Cell Physiol. 22:867-880.

Parvin, S., Lee, O.R., Sathiyaraj, G., Khorolragchaa, A., Kim, Y. 2012. Interrelationship between calmodulin $(\mathrm{CaM})$ and $\mathrm{H}_{2} \mathrm{O}_{2}$ in abscisic acid-induced antioxidant defense in the seedlings of Panax ginseng. Mol. Bio. Rep. 39:7327-7338.

Shan, C., Liang, Z. 2010. Jasmonic acid regulates ascorbate and glutathione metabolism in Agropyron cristatum leaves under water stress. Plant Sci. 178:130-139.

Shan, C., Zhang, S., Li, D., Zhao, Y., Tian, X., Zhao, X., Wu, Y., Wei, X., Liu, R. 2011. Effects of exogenous hydrogen sulfide on the ascorbate and glutathione metabolism in wheat seedlings leaves under water stress. Acta Physiol. Plant. 33:2533-2540.

Shan, C., Zhou, Y., Liu, M. 2015. Nitric oxide participates in the regulation of the ascorbate-glutathione cycle by exogenous jasmonic acid in the leaves of wheat seedlings under drought stress. Protoplasma 252:1-9.

Wang, L., Hou, Z., Hou, L., Zhao, F., Liu, X. 2012. $\mathrm{H}_{2} \mathrm{~S}$ induced by $\mathrm{H}_{2} \mathrm{O}_{2}$ mediates drought-induced stomatal closure in Arabidopsis thaliana. Chin. Bull. Bot. 47:217-225. (in Chinese)

Zhang, A., Jiang, M., Zhang, J., Ding, H., Xu, S., Hu, X., Tan, M. 2007. Nitric oxide induced by hydrogen peroxide mediates abscisic acid-induced activation of the mitogen-activated protein kinase cascade involved in antioxidant defense in maize leaves. New Phytol. 175:36-50. 
Zhang, H., Hu, L.Y., Hu, K.D., He, Y.D., Wang, S.H., Luo, J.P. 2008. Hydrogen sulfide promotes wheat seed germination and alleviates the oxidative damage against copper stress. J. Integr. Plant Biol. 50:15181529.

Zhao, L.Q., Zhang, F., Guo, J.K., Yang, Y.L., Li, B.B., Zhang, L.X. 2004. Nitric oxide functions as a signal in salt resistance in the calluses from two ecotypes of reed. Plant Physiol. 134:849-857. 